\title{
Robustness Analysis and Synthesis for Nonlinear Uncertain Systems
}

\author{
Wei-Min Lu, Member, IEEE, and John C. Doyle
}

\begin{abstract}
A state-space characterization of robustness analysis and synthesis for nonlinear uncertain systems is proposed. The robustness of a class of nonlinear systems subject to $\mathcal{L}_{2}$-bounded structured uncertainties is characterized in terms of a nonlinear matrix inequality (NLMI), which yields a convex feasibility problem. As in the linear case, scalings are used to find a Lyapunov or storage function that give sufficient conditions for robust stability and performances. Sufficient conditions for the solvability of robustness synthesis problems are represented in terms of NLMI's as well. With the proposed NLMI characterizations, it is shown that the computation needed for robustness analysis and synthesis is not more difficult than that for checking Lyapunov stability; the numerical solutions for robustness problems are approximated by the use of finite element methods or finite difference schemes, and the computations are reduced to solving linear matrix inequalities. Unfortunately, while the development in this paper parallels the corresponding linear theory, the resulting computational consequences are, of course, not as favorable.
\end{abstract}

Index Terms-Computational nonlinear control, nonlinear control systems, nonlinear matrix inequality (NLMI), robust control, robust performance, state space.

\section{INTRODUCTION}

I $\mathrm{N}$ THIS paper, we will consider the analysis and synthesis problems for a class of nonlinear systems subject to $\mathcal{L}_{2^{-}}$ bounded structured uncertainty in a state-space setting. The basic tool for robustness analysis of such uncertain systems is the small-gain theorem or its variations (see for example [35], [10], and [12]). A standard diagram for robustness analysis of an uncertain system is depicted in Fig. 1, where $G$ is the nominal system and $\Delta$ is the uncertainty, which is usually assumed to be strictly bounded by one. A general sufficient condition for robust stability is that the gain of the nominal system is less than or equal to one. Overviews of a variety of results in the linear case can be found in [23], [30], [8], and [36]. In those results, the systems are described in terms of their input-output (I/O) behaviors, and robustness analysis is essentially reduced to gain analysis of the corresponding transfer matrices or I/O operators, but most of the results can

Manuscript received February 24, 1995; revised March 18, 1997. Recommended by Associate Editor, M. A. Dahleh. This work was supported in part by AFOSR. This work was conducted when W.-M. Lu was with the Department of Electrical Engineering, California Institute of Technology, Pasadena, CA 91125 USA and the Jet Propulsion Laboratory, Pasadena, CA 91109 USA

W.-M. Lu is with the Advanced Magnetic Recording Laboratory, IBM Corporation, San Jose, CA 95193 USA.

J. C. Doyle is with the Control and Dynamical Systems/Department of Electrical Engineering, California Institute of Technology, Pasadena, CA 91125 USA.

Publisher Item Identifier S 0018-9286(97)08854-5.

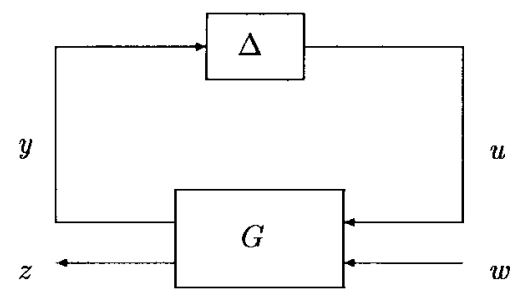

Fig. 1. Uncertain system.

also be expressed using state space [13], [22], [17], [24], [4]. For linear systems, small-gain conditions can be characterized in terms of linear matrix inequalities (LMI's) in state space by the use of the KYP lemma. Therefore, the robustness analysis and synthesis can be conducted by solving LMI's, which are computationally efficient convex feasibility problems (see [4] for a tutorial review of LMI's and their use).

For nonlinear systems, the computational properties of statespace treatment have not been fully exploited yet, although they clearly are much more difficult. In this paper, we will pursue the computational issues further for nonlinear control in state space, attempting to parallel the linear LMI theory as much as possible. We show that the robustness analysis and synthesis for nonlinear systems can also be reduced to the convex feasibility problems with nonlinear matrix inequalities (NLMI's). Note that the state-space treatment captures systems' internal properties, in addition to the I/Obehaviors; therefore, instead of I/O-stability, the notion of asymptotic stability is naturally employed in this paper. By robust asymptotic stability, we mean that the feedback systems are asymptotically stable for each admissible uncertainty; the robust performance means that the uncertain systems are asymptotically stable and have $\mathcal{L}_{2}$-gain $\leq 1$ for each admissible uncertainty.

The robustness results can also be represented in terms of Hamilton-Jacobi equations/inequalities, which is more conventional. However, the NLMI characterizations may offer better computational features. With the NLMI characterization it is shown that computation complexity for checking robustness analysis and synthesis for nonlinear uncertain systems is comparable with that for checking the Lyapunov stability; in other words, the provided approach reduces the robustness analysis and synthesis problems to the possible easiest problems, as far as the computation is concerned. Unfortunately, the NLMI conditions involve neither a finite number of unknowns nor a finite number of constraints, so the computational advantages are far less immediate than for 
LMI's. But the solutions of NLMI's on bounded set can be numerically approximated by the use of finite element method or finite difference spatial discretization; the computations are reduced to solving LMI's. Clearly, much additional work will be needed on the achievement of high computational efficiency, and sophisticated computational techniques will be required to make the general NLMI computation feasible.

In essence, the linear theory fails to fully extend in two ways. First, checking stability for nonlinear systems is much harder so that reducing the computation is less advantageous. Second, the generalization from robust performance analysis to full-information synthesis has serious obstructions, as will be clear. Hopefully, this treatment of the problem will highlight the similarities and differences between the linear and nonlinear cases in a way that will encourage further development of a computational nonlinear control theory.

The rest of the paper is organized as follows. In Section II, some standard results for robustness analysis are reviewed. In particular, the small-gain theorems are given for uncertain systems with structured uncertainty. In Section III, the performance robustness of nonlinear uncertain systems are characterized, in particular, a NLMI characterization is emphasized. In Section IV, we deal with the performance robustness synthesis problem; the solvability conditions are also in terms of NLMI's. We address some computational issues for robustness analysis and synthesis in Section V, in particular, two numerical algorithms are provided, one uses finite element method and the other a finite difference scheme. A numerical example is provided to illustrate the use of finite difference schemes and LMI solvers.

Detailed proofs are omitted throughout because of space constraints. No fundamentally new techniques are introduced, but the interested reader can find more details in [20].

\section{A. Conventions}

The following conventions are made in this paper. $\mathbf{R}$ is the set of real numbers, $\mathbf{R}^{+}:=[0, \infty) \subset \mathbf{R}$. $\mathbf{R}^{n}$ is $n$-dimensional real Euclidean space; $\|\cdot\|$ stands for the Euclidean norm. $\mathbf{X}$ (or $\mathbf{X}_{i}$ ) is the state set which is a convex open subset of some Euclidean space and contains the origin. $\mathbf{R}^{n \times m}\left(\mathbf{C}^{n \times m}\right)$ is the net of all $n \times m$ real (complex) matrices. The transpose of some matrix $M \in \mathbf{R}^{n \times n}$ is denoted by $M^{T}$. By $P \geq 0$ $(P>0)$ for some Hermitian matrix $P \in \mathbf{R}^{n \times n}$ or $\left(\mathbf{C}^{n \times m}\right)$ we mean that the matrix is (semi-)positive definite. A function is said to be of class $\mathbf{C}^{k}$ if it is continuously differentiable $k$ times; so $\mathbf{C}^{0}$ stands for the class of continuous functions. A function $V: \mathbf{X} \rightarrow \mathbf{R}^{+}$is positive-definite if $V(x) \geq 0$, $V(x)=0 \Rightarrow x=0$, and $\lim _{\|x\| \rightarrow \infty} V(x)=\infty$ on $\mathbf{X}$. A $\mathbf{C}^{0}$ matrix-valued function $P: \mathbf{X} \rightarrow \mathbf{R}^{n \times n}$ is positive definite if $P(x)$ is positive definite for each $x \in \mathbf{X}$ and there exist $\alpha>0$ such that $P(x)>\alpha I$ for all $x \in \mathbf{X} . \mathcal{L}_{2}\left(\mathbf{R}^{+}\right)\left(\right.$or $\left.\mathcal{L}_{2}^{p}\left(\mathbf{R}^{+}\right)\right)$ stands for the function space which is defined as the set of all real ( $p$-dimensional) vector-valued functions $u(t)$ on $\mathbf{R}^{+}$ such that $\|u\|_{2}:=\left(\int_{0}^{\infty}\left(\|u(t)\|^{2} d t\right)^{1 / 2}<\infty\right.$, and $\mathcal{L}_{2}^{e}\left(\mathbf{R}^{+}\right)$is its extended space which is defined as the set of the vectorvalued functions $u(t)$ on $\mathbf{R}^{+}$such that $P_{T} u(t) \in \mathcal{L}_{2}\left(\mathbf{R}^{+}\right)$for all $T \in \mathbf{R}^{+}$, where $P_{T}$ is the truncation operator.

\section{PRELIMINARIES: SMALL-Gain \\ THEOREMS AND BOUNDED UNCERTAINTY}

To motivate the techniques to be used in this paper, we will review the small-gain theorems for robust input-output stability. For a causal nonlinear time-invariant operator $G: \mathcal{L}_{2}^{e}[0, \infty) \rightarrow \mathcal{L}_{2}^{e}[0, \infty)$ with $G(0)=0$, the $\mathcal{L}_{2}$-gain is defined as

$$
\|G\|_{\mathcal{L}_{2}}:=\sup _{T \in(0,+\infty)} \sup _{u \in \mathcal{L}_{2}^{e}[0, \infty),\left\|\Gamma_{T} U\right\|_{2} \neq 0} \frac{\left\|P_{T} G u\right\|_{2}}{\left\|P_{T} u\right\|_{2}}
$$

where $P_{T}$ is a truncation operator; $G$ is I/O-stable if $\|G\|_{\mathcal{L}_{2}}<$ $\infty$. $G$ has $\mathcal{L}_{2}$-gain less than or equal to $\gamma \geq 0$ if and only if

$$
\int_{0}^{T}\|y(t)\|^{2} d t \leq \gamma^{2} \int_{0}^{T}\|u(t)\|^{2} d t
$$

for all $u \in \mathcal{L}_{2}^{e}\left(\mathbf{R}^{+}\right)$and $T \in \mathbf{R}^{+}$.

Next, we consider the robust stability of the uncertain system in Fig. 1 without disturbance input $w$ and regulated output $z$, which is denoted by $(G, \Delta)$, where the uncertainty $\Delta \in \Delta$ is structured

$$
\begin{aligned}
\Delta:= & \left\{\text { block-diag }\left[\Delta_{1}, \cdots, \Delta_{N}\right] \mid \Delta_{i}: \mathcal{L}_{2}^{p}[0, \infty)\right. \\
& \left.\rightarrow \mathcal{L}_{2}^{p}[0, \infty) \text { is causal }\right\}
\end{aligned}
$$

and has $\mathcal{L}_{2}$-gain $<1$, i.e., $\Delta \in \mathbf{B} \Delta:=\left\{\Delta \in \Delta \mid\|\Delta\|_{\mathcal{L}_{2}}<\right.$ $1\}$. This description about the uncertainty is fairly general; in many cases, we can use the (frequency-dependent) scaling on both plant and uncertainty, and the problem can be transferred to this standard case. The feedback structure is assumed to be well-posed for any $\Delta \in \mathbf{B} \Delta$, since robust I/O-stability for $(G, \Delta)$ requires that $I-\Delta G$ is stably invertible for all $\Delta \in \mathbf{B} \Delta$

Definition II.1: The structured $\mathcal{L}_{2}$-gain $\mu_{\Delta}(G)$ of $G$ with respect to the structure $\Delta$ in (3) is

$$
\mu_{\Delta}(G):=\sup _{\Delta \in \Delta}\left\{\|\Delta\|_{\mathcal{L}_{2}}^{-1} \mid I-\Delta G \text { is not stably invertible }\right\} .
$$

Otherwise, if $I-\Delta G$ stably invertible for all $\Delta \in \Delta$, then $\mu_{\Delta}(G):=0$.

The uncertain system $(G, \Delta)$ with $\Delta \in \mathbf{B} \Delta$ is thus robustly I/O-stable if and only if $\mu_{\Delta}(G)<1$, but this definition of structured $\mathcal{L}_{2}$-gain does not provide any immediate computational advantage, even for the linear systems. On the other hand, the original uncertain system, $(G, \Delta)$, is equivalent to the scaled system $\left(D G D^{-1}, D \Delta D^{-1}\right)$ where $D \in \mathcal{D}$ defined as

$$
\mathcal{D}:=\left\{\text { block-diag }\left\{d_{1} I, d_{2} I, \cdots, d_{N} I\right\}: d_{i} \in \mathbf{R}, d_{i}>0\right\}
$$

where each of the identity matrices is compatible with the corresponding nonlinear uncertainty $\Delta_{i}$. Note that $\|\Delta\|_{\mathcal{L}_{2}}=$ $\left\|D \Delta D^{-1}\right\|_{\mathcal{L}_{2}}$ for all $D \in \mathcal{D}$ and $\Delta \in \mathbf{B} \Delta$; therefore, for each $D \in \mathcal{D}, \Delta \in \mathbf{B} \Delta$ if and only if $D \Delta D^{-1} \in \mathbf{B} \Delta$. Observe that

$$
\|G\|_{\mathcal{L}_{2}} \geq \inf _{D \in \mathcal{D}}\left\|D G D^{-1}\right\|_{\mathcal{L}_{2}} \geq \mu_{\Delta}(G) .
$$

We immediately have the following scaled small-gain theorem. 
Lemma II.2 (Scaled Small-Gain Theorem): The uncertain system $(G, \Delta)$ is robustly I/O-stable if there is $D \in \mathcal{D}$ such that

$$
\left\|D G D^{-1}\right\|_{\mathcal{L}_{2}} \leq 1
$$

For $D=I$ this is the well-known small-gain theorem (see for example [35]), and (6) suggests why the scaling can reduce the conservatism of the unscaled small-gain condition. The computational implication of the scaled small-gain condition will be discussed in the next sections. Condition (7) is also necessary under some additional assumptions, such as that the plant is linear or nonlinear with fading memory [30], [21], [28]. Similar scaling can be used to reduce the conservatism of analysis of robust performance for the uncertain system Fig. 1 with external disturbance input $w$ and regulated output $z$ against structured perturbations $\Delta$ [12], [36]. Notice that the scaling for reducing the conservatism arising from the constrained structure was previously introduced in conjunction with "interconnected systems stability theory" (see for example [1] and [22]).

In this paper, we will mainly consider the internal properties of the control systems with structured uncertainties; for example, instead of I/O-stability, asymptotic stability is considered in the sequel. We next consider an internal description of the above-bounded uncertainty set. The reader is referred to [33] for a detailed description of dynamical systems with inputs and outputs. Let $\Delta: u \mapsto y$ be a dynamical system with state space $\mathrm{X}$ and initial state $x(0)=0$. It has $\mathcal{L}_{2}$-gain less than or equal to $\gamma$ for some $\gamma>0$ if (2) holds for all $T \geq 0$ and $w(t) \in \mathcal{L}_{2}^{e}\left(\mathbf{R}^{+}\right)$. It is known from [34] and [15] that the system $\Delta$ has $\mathcal{L}_{2}$-gain $\leq 1$ if and only if there exists a nonnegative function $V: \mathbf{X} \rightarrow \mathbf{R}^{+}$, which is known as storage function [34], such that for all $t_{0}, t_{1} \in \mathbf{R}^{+}, x_{0} \in \mathbf{X}$, and $u \in \mathcal{L}_{2}^{p}\left(\mathbf{R}^{+}\right)$the following dissipation inequality holds:

$$
V\left(x_{1}\right)-V\left(x_{0}\right) \leq \int_{t_{0}}^{t_{1}}\left(\|u(t)\|^{2}-\|y(t)\|^{2}\right) d t
$$

where $x_{1}$ is the state for the system with input $u$ at time $t_{1}$. Note that if the storage function is continuously differentiable, then the dissipation inequality (8) becomes

$$
\dot{V}(x) \leq\|u(t)\|^{2}-\|y(t)\|^{2} .
$$

In this paper, we will consider a class $\Omega \subset \Delta$ of the dynamic uncertainties which are $\mathcal{L}_{2}$-gain-bounded.

Definition II.3: The class $\Omega \subset \Delta$ of admissible time-invariant structured uncertainties with $N$ blocks is a set of dynamical systems, and for all $\Delta:=$ block-diag $\left[\Delta_{1}, \cdots, \Delta_{N}\right] \in \Omega$, and for all $i \in\{1, \cdots, N\}$, the dynamic block $\Delta_{i}: \mathcal{L}_{2}^{p_{i}}\left(\mathbf{R}^{+}\right) \rightarrow \mathcal{L}_{2}^{p_{i}}\left(\mathbf{R}^{+}\right)$with state $\xi_{i} \in \mathbf{X}_{i} \subset \mathbf{R}^{d_{i}}$ is asymptotically stable at equilibrium zero with zero input and has that $\mathcal{L}_{2}$-gain $\leq 1$, the corresponding storage function $U_{i}: \mathbf{X}_{i} \rightarrow \mathbf{R}^{+}$, is continuously differentiable.

In the next two sections, we will address the robustness analysis and synthesis problems for nonlinear systems with the above-described admissible structured uncertainty set; the scaling is still used in different analysis and synthesis problems to reduce possible conservatism. Our focus is on the robust performance issues; the robust stability and pure $\mathcal{L}_{2}$-performance are special cases (see [20]).

\section{Robustness ANALYSIS OF UNCERTAIN SySTEMS}

In this section, we will consider the robust performance analysis problem. The feedback uncertain system is represented by block diagram in Fig. 1, where $w$ is some external disturbance vector and $v$ is the regulated signal vector. The nominal plant $G$ has the following realization:

$$
G:\left\{\begin{array}{l}
\dot{x}=f(x)+g_{1}(x) u+g_{2}(x) w \\
y=h_{1}(x)+k_{11}(x) u+k_{12}(x) w \\
z=h_{2}(x)+k_{21}(x) u+k_{22}(x) w
\end{array}\right.
$$

with $x \in \mathbf{X}$, and the uncertainty $\Delta$ is structured which is assumed to belong to the set $\Omega$ defined by Definition II.3. We will assume $k_{11}(x)=0$ for all $x \in \mathbf{X}$ for simplicity, and the nominal system $G$ is strictly causal with respect to the input $u$. The case where $k_{11} \neq 0$ for some $x \in \mathbf{X}$ is considered in [20]. In the following, we denote the uncertain system as $(G, \Delta)$.

Definition III.1: The uncertain system $(G, \Delta)$ depicted in Fig. 1 has robust performance if for each $\Delta \in \Omega$, the corresponding feedback system is well posed and has $\mathcal{L}_{2}$-gain $\leq 1$, i.e.,

$$
\int_{0}^{T}\left(\|z(t)\|^{2}-\|w(t)\|^{2}\right) d t \leq 0
$$

for all $T \in \mathbf{R}^{+}$; in addition, it is asymptotically stable around zero for $w=0$.

In the following we will examine under what conditions the uncertain system above has robust performance. The scaling is used to reduce possible conservatism arising from the structural constraints of the uncertainty; the equivalent scaled system

$$
\left(\left[\begin{array}{cc}
D & 0 \\
0 & I
\end{array}\right] G\left[\begin{array}{cc}
D^{-1} & 0 \\
0 & I
\end{array}\right], D \Delta D^{-1}\right)
$$

will be used to derive the solutions, where $D \in \mathcal{D}$ with the scaling matrix set $\mathcal{D}$ defined in (5). Denoting

$$
\begin{aligned}
& g(x):=\left[\begin{array}{ll}
g_{1}(x) & g_{2}(x)
\end{array}\right], \quad h(x):=\left[\begin{array}{l}
h_{1}(x) \\
h_{2}(x)
\end{array}\right] \\
& k(x):=\left[\begin{array}{ll}
k_{11}(x) & k_{12}(x) \\
k_{21}(x) & k_{22}(x)
\end{array}\right]
\end{aligned}
$$

we have the following result.

Theorem III.2: Consider the uncertain system $(G, \Delta)$ with $G$ is described in (9) and $\Delta \in \Omega$ defined by Definition II.3. The system has robust performance if there exist a positive definite function $V: \mathbf{X} \rightarrow \mathbf{R}$ and a positive definite matrix $\hat{Q} \in \mathcal{D}$ such that any of the following statements is true with $Q:=\left[\begin{array}{cc}\hat{Q} & 0 \\ 0 & I\end{array}\right]$.

1) $Q-k^{T}(x) Q k(x)>0$ and the Hamilton-Jacobi inequality holds as in (10), shown at the bottom of the next page, for all $x \in \mathbf{X} \backslash\{0\}$.

2) Inequality (11), also shown at the bottom of the next page, holds for all $x \in \mathbf{X} \backslash\{0\}$. 
In fact, statements 1) and 2) are equivalent from the Schur complements argument. With some algebraic manipulations, Statement 2) [therefore 1)] implies that the scaled system

$$
\left[\begin{array}{cc}
Q^{1 / 2} & 0 \\
0 & I
\end{array}\right] G\left[\begin{array}{cc}
Q^{-1 / 2} & 0 \\
0 & I
\end{array}\right]
$$

has $\mathcal{L}_{2}$-gain $\leq 1$. Note that the left-hand side of (11) is affine in $V(x)$, thus (11) is actually a differential linear (or affine) matrix inequality, but we will refer to it as NLMI to emphasize its use in nonlinear problems and that they must hold for each $x$. The proof of the theorem is given in [20].

Though the two characterizations in this theorem are equivalent, the NLMI characterization (11) makes immediate some appealing convexity properties: the solutions of NLMI (11) form a convex set. (By the equivalence between the two statements, the solutions of the Hamilton-Jacobi inequality (10) also form a convex set, although it is not immediate and easy to employed in computation. The convexity is a necessary condition for the Hamilton-Jacobi inequality to be equivalently transferred to an NLMI representation.) This trivial fact of the convexity has only been exploited systematically in the linear computation, but we hope that numerical techniques may be developed to exploit it in the nonlinear case as well, although this case is obviously much more difficult. In Section IV, we will consider the numerical issues.

\section{ROBUST SYNTHESIS FOR UNCERTAIN SYSTEMS}

In the last section, robustness of uncertain systems is characterized in terms of NLMI's as a small-gain condition for a (scaled) nominal systems modulo some appropriate stabilizing conditions. Therefore, robust synthesis can combine the analysis results in the last two sections with the techniques of $\mathcal{H}_{\infty}$-control synthesis (see for example [31], [2], and [19]). Technically, we will closely follow the treatments in [19] and [18]. Note that the robust stabilization with unstructured uncertainty is also considered in [32].

Consider the uncertain system Fig. 1 plus additional control input vector $u_{c}$ and measured output vector $y$. As in the analysis problem, $w$ is the external disturbance vector, and $z$ is the regulated signal vector. The nominal plant for robustness synthesis has the following realization:

$$
G:\left\{\begin{array}{l}
\dot{x}=f(x)+g_{1}(x) u+g_{2}(x) w+g_{3}(x) u_{c} \\
y=h_{1}(x)+k_{11}(x) u+k_{12}(x) w+k_{13}(x) u_{c} \\
z=h_{2}(x)+k_{21}(x) u+k_{22}(x) w+k_{23}(x) u_{c}
\end{array}\right.
$$

where $x$ is the state vector of the nominal system, $f, g_{i}, h_{j}$, $k_{i j} \in \mathbf{C}^{0}$, and $f(0)=0, h_{j}(0)=0$, for $i, j=1,2,3$. The uncertainty $\Delta$ is structured and belongs to the set $\Omega$ defined by Definition II.3. Correspondingly, the scaling matrix set $\mathcal{D}$ is defined as (5). In this section, we only consider the state feedback case, i.e., the full state vector $x$ of the nominal system is available for the output measurement. The synthesis problem is stated as follows.

Definition IV.1 (State Feedback Synthesis Problem): Decide when a state feedback law $u_{c}=K(x)$ with $K \in \mathbf{C}^{0}$ and $K(0)=0$ exists for the uncertain system such that the closedloop uncertain system satisfies robust performance.

In the following, we consider the solutions to the above robustness synthesis problem.

\section{A. State Feedback Solutions}

Consider the uncertain system with the nominal plant (12). Define

$$
\begin{gathered}
g(x):=\left[\begin{array}{ll}
g_{1}(x) & g_{2}(x)
\end{array}\right], \quad h(x):=\left[\begin{array}{l}
h_{1}(x) \\
h_{2}(x)
\end{array}\right] \\
k_{1}(x):=\left[\begin{array}{ll}
k_{11}(x) & k_{12}(x) \\
k_{21}(x) & k_{22}(x)
\end{array}\right], \quad k_{2}(x):=\left[\begin{array}{l}
k_{13}(x) \\
k_{23}(x)
\end{array}\right] .
\end{gathered}
$$

The following structural constraints are imposed for simplicity.

Assumption IV.2: $k_{1}(x)=0$, and $k_{2}^{T}(x)\left[h(x) \quad k_{2}(x)\right]=$ [0 $\left.R_{0}(x)\right]$ where $R_{0}(x)>0$ for all $x \in \mathbf{X}$.

Theorem IV.3: Under Assumption IV.2, the state feedback robust performance synthesis problem has a solution for the uncertain system depicted as in Fig. 1 plus control input if there exist a positive definite $\mathbf{C}^{1}$ positive definite function $V: \mathbf{X} \rightarrow \mathbf{R}^{+}$and a positive definite matrix $\hat{Q} \in \mathcal{D}$ such that the following Hamilton-Jacobi inequality holds for all $x \in \mathbf{X} \backslash\{0\}:$

$$
\begin{aligned}
\frac{\partial V}{\partial x}(x) f(x)+ & \frac{1}{4} \frac{\partial V}{\partial x}(x)\left(g(x) Q^{-1} g^{T}(x)-g_{3}(x)\right. \\
& \left.\cdot R_{0}^{-1}(x) g_{3}^{T}(x)\right) \frac{\partial V^{T}}{\partial x}(x)+h^{T}(x) Q h(x)<0
\end{aligned}
$$

where

$$
Q=\left[\begin{array}{ll}
\hat{Q} & 0 \\
0 & I
\end{array}\right]
$$

for all $x \in \mathbf{X} \backslash\{0\}$. Moreover, if $(V(x), \hat{Q})$ is such a pair of solutions, then the state feedback function

$$
K(x)=-\frac{1}{2} R_{0}^{-1}(x) g_{3}^{T}(x) \frac{\partial V}{\partial x}(x)
$$

makes the closed-loop system have a robust performance.

$$
\begin{gathered}
\hat{\mathcal{H}}\left(\frac{\partial V}{\partial x}, Q, x\right):=\frac{\partial V}{\partial x}(x) f(x)+h^{T}(x) Q h(x)+\left(\frac{1}{2} \frac{\partial V}{\partial x}(x) g(x)+h^{T}(x) Q k(x)\right) \\
\cdot\left(Q-k^{T}(x) Q k(x)\right)^{-1}(x) \cdot\left(\frac{1}{2} g^{T}(x) \frac{\partial V^{T}}{\partial x}(x)+k^{T}(x) Q h(x)\right)<0 \\
\mathcal{M}_{R P}\left(\frac{\partial V}{\partial x}, Q, x\right):=\left[\begin{array}{cc}
\frac{\partial V}{\partial x}(x) f(x)+h^{T}(x) Q h(x) & \frac{1}{2} \frac{\partial V}{\partial x}(x) g(x)+h^{T}(x) Q k(x) \\
\frac{1}{2} g^{T}(x) \frac{\partial V^{T}}{\partial x}(x)+k^{T}(x) Q h(x) & k^{T}(x) Q k(x)-Q
\end{array}\right]<0
\end{gathered}
$$


The proof follows the argument in [18] and Theorem III.2; it is given in [20]. However, the computational implication of the Hamilton-Jacobi inequality (13) is not immediate. Note that the Hamilton-Jacobi inequality has unknowns $V$ and $\hat{Q}$; essentially, its left-hand side is not convex in either unknowns, thus it cannot be turned into an NLMI as in Theorem III.2. In the following, we will give an alternative structured characterization with better computational properties.

\section{B. A Convex Characterization for State Feedback Solutions}

Consider the uncertain system with nominal plant (12) with $f$ and $h_{i}$ being sufficiently smooth such that the nominal plant is equivalently represented by the following differential equation:

$$
G:\left\{\begin{array}{l}
\dot{x}=A(x) x+B_{1}(x) u+B_{2}(x) w+B_{3}(x) u_{c} \\
y=C_{1}(x) x+D_{11}(x) u+D_{12}(x) w+D_{13}(x) u_{c} \\
z=C_{2}(x) x+D_{21}(x) u+D_{22}(x) w+D_{23}(x) u_{c}
\end{array}\right.
$$

where $A(x) x=f(x)$ etc., and $A, B_{i}, C_{j}, D_{i j}$ are $\mathbf{C}^{0}$ matrix-valued functions. Notice that the choice of $A(x)$ and $C_{i}(x)$ is not unique; for example, if $A(x) x=f(x)$, then $(A(x)+E(x)) x=f(x)$ for all matrix-valued function $E(x)$ with $E(x) x=0$. In the following, we assume (14) is proper in a sense to be made precise soon. In addition, we assume that $D_{11}(x)=0$ for all $x \in \mathbf{X}$ for simplicity, which also implies that the uncertain system is well posed. We now define

$$
\begin{aligned}
B(x) & :=\left[\begin{array}{ll}
B_{1}(x) & B_{2}(x)
\end{array}\right], \quad C(x):=\left[\begin{array}{l}
C_{1}(x) \\
C_{2}(x)
\end{array}\right] \\
D(x) & :=\left[\begin{array}{cc}
0 & D_{12}(x) \\
D_{21}(x) & D_{22}(x)
\end{array}\right]
\end{aligned}
$$

and

$$
\tilde{B}(x):=\left[\begin{array}{lll}
B_{3}^{T}(x) & D_{13}^{T}(x) & D_{23}^{T}(x)
\end{array}\right] .
$$

Let $\mathcal{N}(\tilde{B}(x))$ be the distribution on $\mathbf{X}$ which annihilates all of the row vectors of $\tilde{B}(x)$. We have the following characterization for the synthesis problem.

Theorem IV.4: Consider the uncertain system with nominal plant defined as (14). The state feedback robust performance synthesis problem has a solution if there exist a positive definite matrix-valued function $X: \mathbf{X} \rightarrow \mathbf{R}^{n \times n}$ and a positive definite matrix $\hat{Y} \in \mathcal{D}$ such that (15), shown at the bottom of the page, holds for all $x \in \mathbf{X}$ with

$$
Y:=\left[\begin{array}{cc}
\hat{Y} & 0 \\
0 & I
\end{array}\right]
$$

where $B_{\perp}(x)$ is a $\mathbf{C}^{0}$ matrix-valued function on $\mathbf{X}$ such that $\operatorname{span}\left(B_{\perp}(x)\right)=\mathcal{N}(\tilde{B}(x))$ for all $x \in \mathbf{X}$, and $\frac{\partial V}{\partial x}(x)=$ $2 x^{T} X^{-1}(x)$ for some $\mathbf{C}^{1}$ function $V$ on $\mathbf{X}$ with $V(0)=0$.

The proof of the above theorem, which uses a generalized Finsler's theorem [19, Lemma 7.3], follows the arguments in the proof of [19, Th. 4.5] and is given in [20]. Note that the NLMI (15) is affine in the unknowns $X(x)$ and $\hat{Y}$; for this part of the characterization, the computation reduces to convex feasibility problems. However, the overall characterization is not convex in general; this can be seen by the additional nonconvex condition $\frac{\partial V}{\partial x}(x)=2 x^{T} X^{-1}(x)$.

As discussed above, there are infinitely many representations for (12) with the form of (14). A natural question is the following: does there exist a proper representation such that the NLMI (15) gives a solution such that the condition $\frac{\partial V}{\partial x}(x)=2 x^{T} X^{-1}(x)$ is automatically satisfied? Note that under some conditions, e.g., Assumption IV.2, the NLMI (15) is equivalent to a state-dependent Riccati inequality (or equation) with unknowns $P(x)=X^{-1}(x)$ and $Q=Y^{-1}$, which can be also derived from Hamilton-Jacobi inequality (13) by replacing $\frac{\partial V}{\partial x}(x)=2 x^{T} P(x)$. In [16], it is shown that there exists a proper representation such that the solution to the corresponding state-dependent Riccati equation satisfies the additional condition. Note that the state-dependent Riccati equation approach was used in nonlinear regulation problem in [6]. But the Riccati equation characterization does not provide immediate computational benefit to the robustness synthesis problem because of the essential nonconvexity.

\section{NLMI's AND NUMERICAL ALGORITHMS}

The computation for robustness analysis and synthesis involves solving NLMI's. In this section, we examine some properties of NLMI's and exploit them in computations for robustness analysis and synthesis.

\section{A. Structural Properties for Robustness Analysis and Synthesis}

To make the computational properties of NLMI's more explicit for different characterizations, we will take Lyapunov stability analysis as a benchmark and compare robustness analysis and robustness synthesis problems with it.

Consider a system $\dot{x}=f(x)$ with $x \in \mathrm{R}^{n}$ being state vector, $f$ is sufficiently smooth, and $f(0)=0$. From the Lyapunov theorem, the system is Lyapunov stable around the equilibrium zero if and only if there is a $\mathbf{C}^{1}$ positive definite function $V: \mathbf{X} \rightarrow \mathbf{R}^{+}$such that

$$
\frac{\partial V}{\partial x}(x) f(x) \leq 0 .
$$

Note that (16) is also an NLMI; moreover, the NLMI characterizations of robustness analysis and synthesis problems share a common structure with that for Lyapunov stability, i.e., each of the solutions involves solving an algebraic NLMI (or statedependent LMI) and a partial differential equation (PDE). The structures of the solutions are summarized in Table I, where $p: \mathbf{X} \rightarrow \mathbf{R}^{1 \times n}$ with $p(0)=0, X: \mathbf{X} \rightarrow \mathbf{R}^{n \times n}$, and $Q$, $Y \in \mathbf{R}^{p \times p}$ are the unknowns.

$$
\mathcal{M}_{S y n}(X, Y, x):=B_{\perp}^{T}(x)\left[\begin{array}{cc}
A(x) X(x)+X(x) A^{T}(x)+B^{T}(x) Y B(x) & X(x) C^{T}(x)+B(x) Y D^{T}(x) \\
C(x) X(x)+D(x) Y B^{T}(x) & D(x) Y D^{T}(x)-Y
\end{array}\right] B_{\perp}(x)<0
$$


TABLE I

\begin{tabular}{c||c|l}
\hline \multicolumn{1}{c||}{ Problems } & Algebraic NLMIs & \multicolumn{1}{c}{ PDEs } \\
\hline Lyapunov Stability & $(16) p(x) f(x) \leq 0$ & $\frac{\partial V}{\partial x}(x)=p(x)$ \\
\hline Robustness Analysis & $(11) \mathcal{M}_{R P}(p(x), Q, x) \leq 0$ & $\frac{\partial V}{\partial x}(x)=p(x)$ \\
\hline Robustness Synthesis & $(15) \mathcal{M}_{S y n}(X(x), Y, x)<0$ & $\frac{\partial V}{\partial x}(x)=2 x^{T} X^{-1}(x)$ \\
\hline
\end{tabular}

Because of the similarity in solution structures between the robustness analysis/synthesis problems and Lyapunov stability, we conclude that the computational effort needed for robustness analysis and synthesis is not more difficult than that for checking Lyapunov stability. In other words, the NLMI characterization reduces the robustness analysis and synthesis problems to the possible easiest problems in the computational point of view. In the following, we propose two types of numerical algorithms for solving the above analysis and synthesis problems by taking advantage of NLMI characterizations.

\section{B. Finite Element Methods}

Let us first examine the algebraic NLMI's. The algebraic NLMI's for robustness synthesis have square matrix unknowns. The algebraic NLMI's for robustness analysis can also be turned into the same form. Let us take (11) as an example: Let $f(x)=A(x) x, g(x)=B(x), h(x)=C(x) x$, $k(x)=D(x)$, and $p(x)=P(x) x$ where $A, B, C$, and $D$ are properly chosen continuous matrix-valued functions (see [16]), then the solution of (17), as shown at the bottom of the page, can be obtained by the solution of (18), also shown at the bottom of the page. It is sufficient to find a $\mathbf{C}^{\mathbf{0}}$ matrixvalued function $P: \mathbf{X} \rightarrow \mathbf{R}^{n \times n}$ and a positive definite matrix $Q$ such that $\mathcal{M}(P, Q, x)<0$. Therefore, if $(P(x), Q)$ is a solution to (18), then $\left(2 x^{T} P(x), Q\right)$ is a solution to (17). The left-hand side of NLMI (18) or (15) is affine in its unknown, for example

$$
\mathcal{M}\left(\sum_{k=1}^{N} \alpha_{k} P_{k}, \sum_{k=1}^{N} \alpha_{k} Q_{k}, x\right)=\sum_{k=1}^{N} \alpha_{k} \mathcal{M}\left(P_{k}, Q_{k}, x\right)
$$

for all $\alpha_{k} \geq 0$ with $\sum_{k=1}^{N} \alpha_{k}=1$. Equation (19) implies that the (positive definite) $\mathbf{C}^{0}$ solutions $P(x)$ and $Q$ to NLMI $\mathcal{M}(P(x), Q, x)<0$ form a convex set. Using this property, one can also show that the positive definite solutions of the NLMI (18) can always be chosen to be continuous if they exist.

Theorem V.1: Suppose the matrix inequality $\mathcal{M}(P, Q, x)$ $<0$ has positive definite solution pair $\left(P_{x}, Q\right)$, where $P_{x}$ depends on $x \in \mathbf{X} \subset \mathbf{R}^{n}$, then there exists a $\mathbf{C}^{0}$ matrix- valued function $P: \mathbf{X} \rightarrow \mathbf{R}^{n \times n}$ with $P(x)=P^{T}(x)>0$, such that $\mathcal{M}(P(x), Q, x)<0$ for all $x \in \mathbf{X}$.

Proof: Consider the NLMI (18). By assumption, there exist a positive definite matrix pair $Q \in \mathbf{R}^{p \times p}$ and $P_{x} \in \mathbf{R}^{n \times n}$ for each $x \in \mathbf{X}$ such that

$$
\mathcal{M}\left(P_{x}, Q, x\right)<0
$$

By continuity of $M$ with respect to $x$, there is an $r_{x}>0$ such that

$$
\mathcal{M}\left(P_{x}, Q, x_{0}\right)<0
$$

for all $x_{0} \in \mathbf{N}(x):=\left\{x_{0}:\left\|x_{0}-x\right\|<r_{x}\right\}$.

Now $\left.\{\mathbf{N}(x)\}\right|_{x \in \mathbf{X}}$ is an open covering of $\mathbf{X} \subset \mathbf{R}^{n}$, then there is a locally finite open subcovering $\left.\left\{\mathbf{N}_{i}\right\}\right|_{i \in \mathbf{I}}$ for some index set $\mathbf{I}$ which refines $\left.\{\mathbf{N}(x)\}\right|_{x \in \mathbf{X}}$. Now $P_{i} \in \mathbf{R}^{n \times n}$ is positive definite for each $i \in \mathbf{I}$ such that

$$
\mathcal{M}\left(P_{i}, Q, x\right)<0
$$

for all $x \in \mathbf{N}_{i}$.

It is known by the standard argument of partitions of unity that there is a smooth partition of unity $\left.\left\{\phi_{i}\right\}\right|_{i \in \mathbf{I}}$ to $\mathbf{X}$ subordinated to the covering $\left.\left\{\mathbf{N}_{i}\right\}\right|_{i \in \mathbf{I}}$; i.e., $\phi_{i}$ is smooth and nonnegative with support $\operatorname{Supp}\left(\phi_{\mathbf{i}}\right) \subset \mathbf{N}_{\mathbf{i}}$ for each $i \in \mathbf{I}$, and

$$
\sum_{i \in \mathbf{I}} \phi_{i}(x)=1, \quad \forall x \in \mathbf{X} .
$$

Define $P: \mathbf{X} \rightarrow \mathbf{R}^{n \times n}$ as

$$
P(x)=\sum_{i \in \mathbf{I}} \phi_{i}(x) P_{i}, \quad \forall x \in \mathbf{X}
$$

which is positive definite for all $x \in \mathbf{X}$ and smooth since it is locally a finite sum of smooth positive definite matrix-valued functions.

It follows from (22), (23), and (19) that

$$
\begin{aligned}
\mathcal{M}(P(x), Q, x) & =\mathcal{M}\left(\sum_{i \in \mathbf{I}} \phi_{i}(x) P_{i}, Q, x\right) \\
& =\sum_{i \in \mathbf{I}} \phi_{i}(x) \mathcal{M}\left(P_{i}, Q, x\right)<0 .
\end{aligned}
$$

$$
\begin{aligned}
\mathcal{M}_{R P}(p, Q, x) & :=\left[\begin{array}{cc}
p(x) f(x)+h^{T}(x) Q h(x) & \frac{1}{2} p(x) g(x)+h^{T}(x) Q k(x) \\
\frac{1}{2} g^{T}(x) p^{T}(x)+k^{T}(x) Q h(x) & k^{T}(x) Q^{k}(x)-Q
\end{array}\right] \leq 0 \\
\mathcal{M}(P, Q, x): & =\left[\begin{array}{cc}
A^{T}(x) P(x)+P^{T}(x) A(x)+C^{T}(x) Q C(x) & P^{T}(x) B(x)+C^{T}(x) Q D(x) \\
B^{T}(x) P(x)+D^{T}(x) Q C(x) & D^{T}(x) Q D(x)-Q
\end{array}\right]<0
\end{aligned}
$$


Therefore, the constructed smooth positive definite matrixvalued function $P: \mathbf{X} \rightarrow \mathbf{R}^{n \times n}$ in (23) is a solution to $\mathcal{M}(P(x), Q, x)<0$.

The NLMI (18) or (15) is actually a state-dependent LMI on $\mathbf{X}$. In general cases where the state set $\mathbf{X}$ is not finite, the computation for solving NLMI's is an infinite-dimensional LMI problem. Though this is bad news for computational efforts to deal with nonlinear problems, some approximation computation methods can still be used. In fact, the constructive proof of the above theorem suggests a finite element method to numerically approximate solutions of algebraic NLMI (18) or (15) on a bounded state set $\mathbf{X} \subset \mathbf{R}^{n}$.

To illustrate the finite element ideas, let open sets $N_{i}$ $(i=1, \cdots, I)$ be a finite covering of $\mathbf{X}$, i.e.,

$$
\mathbf{X}=\bigcup_{i=1}^{I} N_{i}
$$

Choose the trial functions to be a partition of unity $\left\{\phi_{i}\right\}$ to $\mathbf{X}$ subordinated to the covering $\left\{N_{i}\right\}$. Then the solution $P(x)$ of algebraic NLMI (18) or (15) for $x \in \mathbf{X}$ can be approximated by the following finite expansion:

$$
P(x)=\sum_{i=1}^{I} \phi_{i}(x) P_{i}, \quad \forall x \in \mathbf{X}
$$

where the coefficient matrix $P_{i}$ is chosen to satisfy the following set of coupled LMI's:

$$
\mathcal{M}\left(P_{i}, Q, x_{i}\right)<0
$$

for some $x_{i} \in N_{i}$, where $P_{i}$ and $Q$ are unknowns.

The above finite element algorithm indirectly gives the numerical solutions to the robustness analysis or synthesis problems. However, even if the algorithm happens to satisfy

$$
\mathcal{M}\left(P_{i}, Q, x\right)<0
$$

for all $x \in N_{i}, p(x)=2 x^{T} P(x)$ [or $p(x)=2 x^{T} X^{-1}(x)$ ] still does not give the exact solutions to the original analysis or synthesis problems. It is additionally required that there be a $\mathbf{C}^{1}$ function $V: \mathbf{X} \rightarrow \mathbf{R}^{+}$such that

$$
\frac{\partial V}{\partial x}(x)=p^{T}(x)
$$

for all $x \in \mathbf{X}$. The finite element algorithm does not guarantee (25). Thus, the above finite element method only gives approximated solutions. There are more discussions about (25) in the context of NLMI solution in [20]. In the next subsection, we will give another numerical algorithm where (25) is integrated into the algorithm.

\section{Finite Difference Schemes}

The finite difference scheme is a useful (recursive) numerical method of finding approximated solutions to PDE's, in particular Hamilton-Jacobi equations [7], where the difference approximation technique is used to approximate partial derivatives. The finite difference technique can be naturally employed to numerically approximate the solutions of NLMI's arising from the robustness analysis problems, e.g., (26), shown at the bottom of the page, on $\mathbf{X} \subset \mathbf{R}^{n}$, with $V(0)=0$.

To implement the finite-difference algorithm, we first grid the state space $\mathbf{R}^{n}$. A generic point in $\mathbf{R}^{n}$ will be written as $\left(x_{1}, \cdots, x_{n}\right)$. Let the mesh sizes be $\Delta x_{i}>0$ for $i=1,2, \cdots, n$. Let $V_{\left(i_{1}, i_{2}, \ldots, i_{n}\right)}$ be the value of numerical approximation of $V(x)$ at $\left(i_{1} \Delta x_{1}, \cdots, i_{n} \Delta x_{n}\right)$ for $i_{1}, \cdots, i_{n} \in \mathbf{Z}$. The gradient $\frac{\partial V}{\partial x}$ at $\left(i_{1} \Delta x_{1}, \cdots, i_{n} \Delta x_{n}\right)$ can be approximated by the following difference formula:

$$
\begin{aligned}
\frac{\partial V}{\partial x}\left(i_{1} \Delta x_{1}, \cdots, i_{n} \Delta x_{n}\right) & \\
\approx & {\left[\frac{V_{\left(i_{1}+1, i_{2}, \cdots, i_{n}\right)}-V_{\left(i_{1}, i_{2}, \cdots, i_{n}\right)}}{\Delta x_{1}},\right.} \\
& \left.\cdots, \frac{V_{\left(i_{1}, i_{2}, \cdots, i_{n}+1\right)}-V_{\left(i_{1}, i_{2}, \cdots, i_{n}\right)}}{\Delta x_{n}}\right] .
\end{aligned}
$$

By substituting the difference approximation, the "differenced form" of (26) at $x=\left(i_{1} \Delta x_{1}, \cdots, i_{n} \Delta x_{n}\right)$ becomes (28), also shown at the bottom of the page, with $V_{(0,0, \cdots, 0)}=0$. Inequality (28) is an LMI, because the left-hand side of the inequality is affinite in $V_{\left(i_{1}+1, i_{2}, \ldots, i_{n}\right)}$, $V_{\left(i_{1}, i_{2}+1, \cdots, i_{n}\right)}, \cdots, V_{\left(i_{1}, i_{2}, \cdots, i_{n}+1\right)}$, and $V_{\left(i_{1}, i_{2}, \cdots, i_{n}\right)}$. Therefore, the nonnegative $V_{\left(i_{1}, i_{2}, \cdots, i_{n}\right)}$ for each $\left(i_{1}, i_{2}, \cdots, i_{n}\right)$ can be computed by solving the above set of LMI's for all $i_{1}, \cdots, i_{n}$. If the computational memory is a concern, an iteration approach can be used instead; the solutions at each iteration involves solving the LMI (28) with unknown, say, $V_{\left(i_{1}+1, \cdots, i_{n}\right)}$ etc., being nonnegative where $V_{\left(i_{1}, i_{2}, \cdots, i_{n}\right)}$ etc. are known.

Observe that the computational complexity of finitedifference method is exponentially dependent on the dimension of the state vector. However, the advantage of using the finite difference method in NLMI computation is that the computations are reduced to solving LMI's, which are convex feasibility problems. The finite difference method with NLMI characterization is more computationally efficient than that with Hamilton-Jacobi equation characterization. For example, consider instead the corresponding Hamilton-Jacobi equation:

$$
\mathcal{H}\left(\frac{\partial V}{\partial x}(x), x\right)=0, \quad V(0)=0
$$

$$
\mathcal{M}\left(\frac{\partial V}{\partial x}(x), x\right):=\left[\begin{array}{cc}
\frac{\partial V}{\partial x}(x) f(x)+h^{T}(x) h(x) & \frac{1}{2} \frac{\partial V}{\partial x}(x) g(x)+h^{T}(x) k(x) \\
\frac{1}{2} g^{T}(x) \frac{\partial V^{T}}{\partial x}(x)+k^{T}(x) h(x) & k^{T}(x) k(x)-I
\end{array}\right] \leq 0
$$

$$
\mathcal{M}\left(\left[\frac{V_{\left(i_{1}+1, i_{2}, \cdots, i_{n}\right)}-V_{\left(i_{1}, i_{2}, \cdots, i_{n}\right)}}{\Delta x_{1}}, \cdots, \frac{V_{\left(i_{1}, i_{2}, \cdots, i_{n}+1\right)}-V_{\left(i_{1}, i_{2}, \cdots, i_{n}\right)}}{\Delta x_{n}}\right],\left(i_{1} \Delta x_{1}, \cdots, i_{n} \Delta x_{n}\right)\right) \leq 0
$$




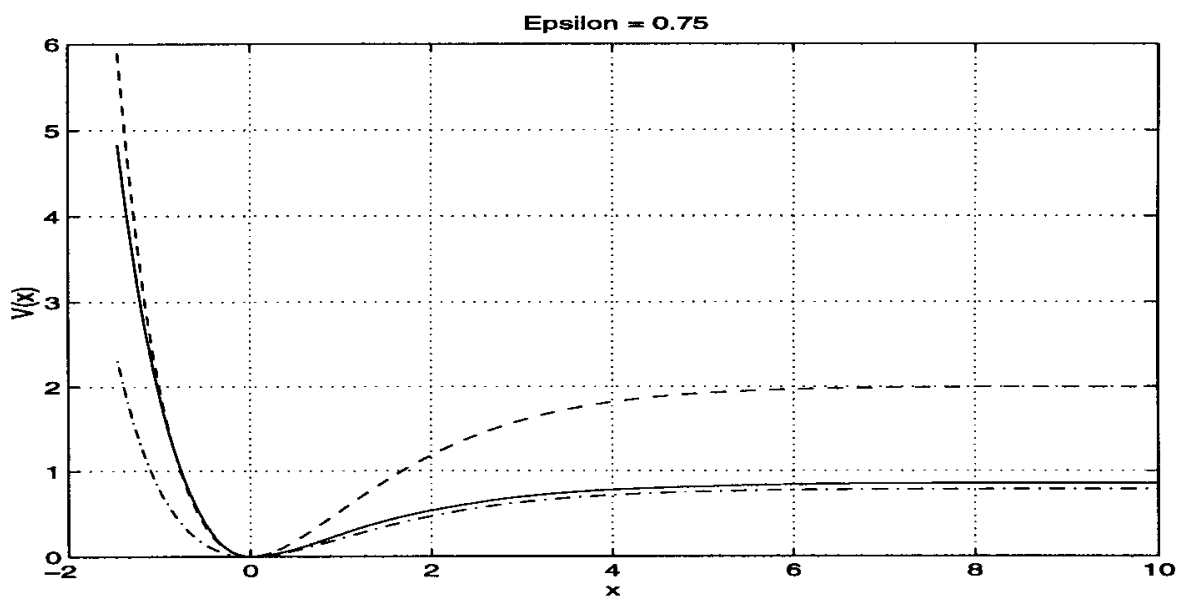

Fig. 2. Numerical solution of NLMI example.

with $x \in \mathbf{R}^{n}$ with $V(0,0)=0$. To use the finite difference method, we similarly grid the state space; the gradient $\frac{\partial V}{\partial x}$ can be similarly approximated as partial difference in (27). Therefore, the Hamilton-Jacobi equation (29) is approximated by the following difference equation:

$$
\begin{array}{r}
\mathcal{H}\left(\left[\frac{V_{\left(i_{1}+1, \cdots, i_{n}\right)}-V_{\left(i_{1}, \cdots, i_{n}\right)}}{\Delta x_{1}}, \cdots, \frac{V_{\left(i_{1}, \cdots, i_{n}+1\right)}-V_{\left(i_{1}, \cdots, i_{n}\right)}}{\Delta x_{n}}\right]\right. \\
\left.\left(i_{i} \Delta x_{1}, \cdots, i_{n} \Delta x_{n}\right)\right)=0 .
\end{array}
$$

One needs to solve the implicit nonlinear equation to get the approximated solutions, $V_{\left(i_{1}, \cdots, i_{n}\right)}$ for all $\left(i_{1}, \cdots, i_{n}\right)$. There are several numerical methods to solve the above nonlinear equation, such as Jacobi and Gauss-Seidel methods [3]. For example, if $V_{\left(i_{1}, \cdots, i_{n}\right)}^{k}$ for each $\left(i_{1}, \cdots, i_{n}\right)$ in the following iteration:

$$
\begin{aligned}
& V_{\left(i_{1}, \cdots, i_{n}\right)}^{k+1}=V_{\left(i_{1}, \cdots, i_{n}\right)}^{k}-V_{\left(i_{1}, \cdots, i_{n}\right)}^{k} \\
& -\lambda \mathcal{H}\left(\left[\frac{V_{\left(i_{1}+1, \cdots, i_{n}\right)}^{k}}{\Delta x_{1}}\right]\right. \\
& \left.\left.\quad \cdots, \frac{V_{\left(i_{1}, \cdots, i_{n}+1\right)}^{k}-V_{\left(i_{1}, \cdots, i_{n}\right)}^{k}}{\Delta x_{n}}\right],\left(i_{i} \Delta x_{1}, \cdots, i_{n} \Delta x_{n}\right)\right)
\end{aligned}
$$

converges for an appropriately chosen $\lambda \neq 0$, then

$$
\lim _{k \rightarrow \infty} V_{\left(i_{1}, \cdots, i_{n}\right)}^{k}=V_{\left(i_{1}, \cdots, i_{n}\right)}
$$

is the numerical solution to the original Hamilton-Jacobi equation.

To conclude this section, we will give a numerical example to illustrate the use of the finite difference scheme in NLMI computation.

Example V.2: Consider the following two-input-singleoutput system:

$$
\left\{\begin{array}{l}
\dot{x}=-e^{x} x+\left[\begin{array}{ll}
-e^{x} & e^{x}
\end{array}\right] w \\
z=\epsilon x+\left[\begin{array}{ll}
\epsilon & 0
\end{array}\right] w
\end{array}\right.
$$

To verify that the system has a performance with $\mathcal{L}_{2}$-gain $\leq \frac{1}{\epsilon}$ for $0<\epsilon<1$, we consider the solutions of the corresponding
NLMI as follows:

$\left[\begin{array}{ccc}-\frac{\partial V}{\partial x}(x) e^{x} x+\epsilon^{2} x^{2} & -\frac{1}{2} \frac{\partial V}{\partial x}(x) e^{x}+\epsilon^{2} x & \frac{1}{2} \frac{\partial V}{\partial x}(x) e^{x} \\ -\frac{1}{2} \frac{\partial V}{\partial x}(x) e^{x}+\epsilon^{2} x & \epsilon^{2}-1 & 0 \\ \frac{1}{2} \frac{\partial V}{\partial x}(x) e^{x} & 0 & -1\end{array}\right] \leq 0$.

The exact solution $V(x)$ can be represented as $V(x)=$ $x^{2} \int_{0}^{1} t P(t x) d t$, where $P(x)$ satisfies $\frac{2 \epsilon^{2}}{2-\epsilon^{2}} e^{-x} \leq P(x) \leq$ $2 e^{-x}$; therefore, $V(x) \geq 0$ must satisfy

$$
\frac{2 \epsilon^{2}}{2-\epsilon^{2}}\left(1-(1+x) e^{-x}\right) \leq V(x) \leq 2\left(1-(1+x) e^{-x}\right) .
$$

(See Fig. 2 for $\epsilon=3 / 4$, where the solution range is bounded by the dashed lines.) Next, the finite difference (recursive scheme described above) is used to find numerical solutions of NLMI (30) on $[-2,10]$ with $\epsilon=3 / 4$. At each iterative step, an LMI is solved using LMI-toolbox [14]. The mesh size is varying between 0.001 and 0.1 . The numerical solution of $V(x)$ is shown in Fig. 2 as the solid line, which is in the solution range.

\section{ACKNOWLEDGMENT}

The authors would like to thank Y. Huang for her help with the implementation of the finite difference algorithm in Example V.2.

\section{REFERENCES}

[1] M. Araki, "Stability of large-scale-systems: Quadratic-Order theory of composite-systems method using $M$-matrices," IEEE Trans. Automat. Contr., vol. AC-23, pp. 129-142, 1978.

[2] J. A. Ball, J. W. Helton, and M. L. Walker, " $\mathcal{H}_{\infty}$ control for nonlinear systems with output feedback," IEEE Trans. Automat. Contr., vol. 38, pp. 546-559, 1993.

[3] D. P. Bertsekas and J. N. Tsitsiklis, Parallel and Distributed Computation: Numerical Methods. Englewood Cliffs, NJ: Prentice-Hall, 1989.

[4] S. P. Boyd, L. El Ghaoui, E. Feron, and V. Balakrishnan, Linear Matrix Inequalities in System and Control Theory. Philadelphia, PA: SIAM, 1994.

[5] M. J. Chen and C. A. Desoer, "Necessary and sufficient condition for robust stability of linear distributed feedback systems," Int. J. Contr., vol. 35, pp. 255-267, 1982.

[6] J. R. Cloutier, C. N. D'Souza, and C. P. Mracek, "Nonlinear regulation and nonlinear $\mathcal{H}_{\infty}$-control via the state-dependent Riccati equation technique," in Proc. 1st Int. Conf. Nonl. Problems Aviat. Aerospace, Daytona Beach, FL, May 1996. 
[7] M. G. Crandall and P. L. Lions, "Two approximations of solutions of Hamilton-Jacobi equations," Math. Computation, vol. 43, pp. 1-19, 1984.

[8] M. A. Dahleh and I. J. Diaz-Bobillo, Control of Uncertain Systems. Prentice-Hall, 1995

[9] M. A. Dahleh and M. H. Khammash, "Controller design for plants with structured uncertainty," Automatica, vol. 29, pp. 37-56, 1993.

[10] J. C. Doyle, "Analysis of feedback systems with structured uncertainties," Proc. Inst. Elec. Eng., vol. 129, pt. D, pp. 242-250, 1982.

[11] J. C. Doyle and G. Stein, "Multivariable feedback design: Concepts for a classical/modern synthesis," IEEE Trans. Automat. Contr., vol. AC-26, pp. 4-16, 1981

[12] J. C. Doyle, J. E. Wall, and G. Stein, "Performance and robustness analysis for structured uncertainty," in Proc. 21st IEEE Conf. Decision Contr., 1982, pp. 629-636.

[13] J. C. Doyle, K. Glover, Khargonekar, and B. Francis, "State-Space solutions to standard $\mathcal{H}_{2}$ and $\mathcal{H}_{\infty}$ control problems," IEEE Trans. Automat. Contr., vol. 34, pp. 831-847, 1989.

[14] P. Gahinet, A. Nemirovskii, A. J. Laub, and M. Chilali, "The LMI control toolbox," in Proc. IEEE Conf. Decision Contr., Orlando, FL, 1994, pp. 2038-2041.

[15] D. J. Hill and P. J. Moylan, "Dissipative dynamical systems: Basicinput-output and state properties," J. Franklin Inst., vol. 30, no. 5, pp. $1154-1170,1980$

[16] Y. Huang and W. M. Lu, "Nonlinear optimal control: Alternatives to Hamilton-Jacobi equation," in Proc. IEEE Conf. Decision Contr., Kobe, Japan, Dec. 1996.

[17] W.-M. Lu, K. Zhou, and J. C. Doyle, "Stabilization of linear uncertain systems: An LFT approach," IEEE Trans. Automat. Contr., vol. 41, pp. $1-15,1996$

[18] W.-M. Lu and J. C. Doyle, "H-Ho-Control of nonlinear systems: A class of controllers," Caltech CDS Tech. Memo., CIT-CDS-93-008; also in Proc. 32nd IEEE Conf. Decision Contr., San Antonio, TX, 1993, pp. $166-171$.

[19] _ " $\mathcal{H}_{\infty}$-Control of nonlinear systems: A convex characterization," IEEE Trans. Automat. Contr., vol. 40, pp. 1668-1675, 1995.

[20] _ "Robustness analysis and synthesis of nonlinear uncertain systems," Caltech CDS Tech. Memo., 1997; also "A state-space approach to robustness analysis and synthesis of nonlinear uncertain systems," Caltech CDS Tech. Memo., CIT-CDS-94-010, 1994.

[21] A. Megretski, "Necessary and sufficient conditions of stability: A multiloop generalization of circle criterion," IEEE Trans. Automat. Contr., vol. 38, pp. 753-756, 1993

[22] P. J. Moylan and D. J. Hill, "Stability criteria for large-scale systems," IEEE Trans. Automat. Contr., vol. AC-23, pp. 143-149, 1978.

[23] A. Packard and J. C. Doyle, "The complex structured singular value," Automatica, vol. 29, pp. 71-109, 1993.

[24] A. Packard, K. Zhou, P. Pandey, and G. Becker, "A collection of robust control problems leading to LMI's," in Proc. 1991 IEEE Conf. Decision Contr., Brighton, U.K., pp. 1245-1250.

[25] A. Packard and M. Kantner, "Nonlinear control the LPV way," in Proc. IEEE Conf. Decision Contr., Kobe, Japan, 1996.

[26] N. Rouche, P. Habets, and M. Laloy, Stability Theory by Liapunov's Direct Method. New York: Springer-Verlag, 1977.

[27] M. G. Safonov, "Stability margins of diagonally perturbed multivariable feedback systems," in Proc. Inst. Elec. Eng., vol. 129, pt. D, pp. 251-256, 1982

[28] A. V. Savkin and I. R. Petersen, "Nonlinear versus linear control in the absolute stabilizability of uncertain systems with structured uncertainty," in Proc. IEEE Conf. Decision Contr., San Antonio, TX, 1993, pp. $172-177$.

[29] J. S. Shamma, "The necessity of small-gain theorem for time-varying and nonlinear systems," IEEE Trans. Automat. Contr., vol. 36, pp. $1138-1147,1991$.

[30] _ "Robust stability with time-varying structured uncertainty," IEEE Trans. Automat. Contr., vol. 39, pp. 714-724, 1994.
[31] A. J. van der Schaft, " $\mathcal{L}_{2}$-Gain analysis of nonlinear systems and nonlinear state feedback $\mathcal{H}_{\infty}$-control," IEEE Trans. Automat. Contr. vol. 37, pp. 770-784, 1992.

[32] _ "Robust stabilization of nonlinear systems via stable kernel representations with $\mathcal{L}_{2}$-gain bounded uncertainty," Syst. Contr. Lett., vol. 24 , pp. $75-81,1995$.

[33] J. C. Willems, The Analysis of Feedback Systems. Cambridge, MA MIT Press, 1971

[34] _ "Dissipative dynamical systems (Parts I and II)," Arch. Rat. Mech. Anal., vol. 45, pp. 321-393, 1972.

[35] G. Zames, "On the input-output stability of time-varying nonlinear feedback systems: Conditions using concepts of loop gain, conicity, and positivity," IEEE Trans. Automat. Contr., vol. AC-11, pp. 228-238, 1966.

[36] K. Zhou, J. C. Doyle, and K. Glover, Robust and Optimal Control. Upper Saddle River, NJ: Prentice-Hall, 1996.

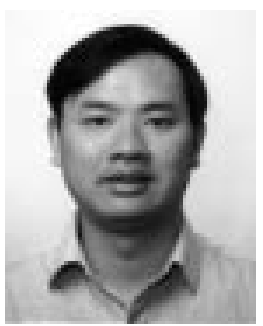

Wei-Min Lu (S'88-M'96) was born in Changde, Hunan Province, China. He received the B.Sc. and M.Sc. degrees in automatic control from the Tsinghua University, Beijing, China, in 1986 and 1989, respectively, and the Ph.D. degree in electrical engineering from the California Institute of Technology, Pasadena, CA, in 1995.

Since 1996, he has been with NASA Jet Propulsion Laboratory (JPL), where he is a member of the technical staff. Before he joined JPL, he had been with UC-Berkeley as a Postdoctoral Research Fellow for a year. His general technical interests are in theory, computation, and application of control science. Currently, his research is focused on robust and adaptive control, theoretical and computational nonlinear control, as well as discrete event and hybrid control; he is particularly interested in the industrial application of control technologies to the design of complex systems with high performance and reliability, including advanced aerospace systems, and information storage systems.

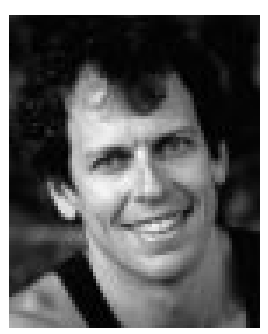

John C. Doyle received the B.S. and M.S. degrees in electrical engineering from the Massachusetts Institute of Technology, Cambridge, in 1977 and the $\mathrm{Ph} . \mathrm{D}$. degree in mathematics from the University of California, Berkeley, in 1984.

$\mathrm{He}$ is currently a Professor of Control and Dynamical Systems and Electrical Engineering at California Institute of Technology. His theoretical research interests include modeling, identification, analysis, and control of uncertain and nonlinear systems, and his work has been applied throughout the aerospace and process control industries. His applications interests are motivated by the interplay between control, dynamical systems, and design and analysis of large, complex engineering systems. Other interests include modeling and control of acute and chronic response to exercise, and feminist critical theory.

Dr. Doyle is the recipient of the Hickernell Award, the Eckman Award, the IEEE Control Systems Society Centennial Outstanding Young Engineer Award, the Bernard Friedman Award, NSF Presidential Award, and ONR Young Investigators Award. He has co-authored papers that have won the AACC Hugo Schuck Award, two IEEE Transactions George S. Axelby Outstanding Paper Awards, the IEEE Baker Prize, as well as papers that have ranked in the top ten "most important" papers worldwide in pure and applied mathematics from 1981 to 1993 in a survey conducted by CTWS-Leiden. 